\title{
Géolinguistique
}

21 | 2021

Varia

\section{Variation des classes motivationnelles parmi les données phyconymiques de la zone celtique (Basse-Bretagne, Irlande)}

Semantic Motivational Variation among Seaweed Names in Celtic

(Lower-Brittany, Ireland)

\section{Mael Jézéquel}

\section{OpenEdition \\ Journals}

Édition électronique

URL : https://journals.openedition.org/geolinguistique/5987

DOI : 10.4000/geolinguistique.5987

ISSN : 2650-8176

Éditeur

UGA Éditions/Université Grenoble Alpes

Édition imprimée

ISBN : 978-2-37747-303-8

ISSN : 0761-9081

Référence électronique

Mael Jézéquel, « Variation des classes motivationnelles parmi les données phyconymiques de la zone celtique (Basse-Bretagne, Irlande) », Géolinguistique [En ligne], 21 | 2021, mis en ligne le 06 décembre 2021, consulté le 19 décembre 2021. URL : http://journals.openedition.org/geolinguistique/5987 DOI : https://doi.org/10.4000/geolinguistique.5987

Ce document a été généré automatiquement le 19 décembre 2021.

Géolinguistique 


\title{
Variation des classes motivationnelles parmi les données phyconymiques de la zone celtique (Basse-Bretagne, Irlande)
}

\author{
Semantic Motivational Variation among Seaweed Names in Celtic \\ (Lower-Brittany, Ireland)
}

Mael Jézéquel

\section{NOTE DE L'ÉDITEUR}

Cette étude s'inscrit dans le cadre de la thèse Aires linguistiques en zone Manche-Atlantique. Concordances entre zones celtiques, romanes et anglo-germaniques du Portugal à la Bretagne et à l'Écosse, dirigée depuis fin 2018 par Daniel Le Bris CRBC-UBO et Xaverio Ballester, département de philologie, université de Valence (Espagne). Elle a obtenu un financement de thèse ARED auprès de la Région Bretagne. Ce travail s'inscrit également depuis 2020 dans le projet ArMeRIE (Archéologie maritime et recherche interdisciplinaire environnementale) dirigé par Yvan Pailler, archéologue INRAP-UBO. $<$ www.armerie.fr>.

\section{Introduction}

Dans son analyse des désignations de l'arc-en-ciel en Europe, Alinei (1983) proposait de distinguer des périodes d'innovations lexicales selon le système de croyance supposé par la motivation du signifiant. Trois strates se succèdent alors selon lui : une première, qui correspondrait à la période zoomorphique ou totémique et impliquant un motif animal pour désigner l'arc-en-ciel, par exemple "baleine ». La deuxième période, dite anthropomorphique, associe le phénomène météorologique à l'image de l'homme, 
exemple «madame Lune ». La plus récente concernerait des désignations motivées par les entités de la religion chrétienne ou islamique comme "arc d'Allah». Loin de pouvoir fournir une datation précise aux innovations lexicales, à la manière d'une analyse isotopique, il proposait de périodiser les désignations selon la façon dont elle témoignait d'une représentation particulière du monde, évolutive sur plusieurs milliers d'années. Cette démarche intervient dans un contexte où les preuves matérielles permettant de parler de religion à la Préhistoire sont minces mais dont la mythologie transmise à l'oral nous amène à considérer le principe de continuité comme base théorique de recherche en ethnolinguistique (Alinei, 1996-2000). À la suite de travaux plus anciens comme ceux de Guiraud (1967) ou plus récents avec Dalbera (2006), de nombreuses autres études ont été menées sous l'impulsion du Centre de dialectologie de Grenoble ${ }^{1}$ notamment dans le cadre de recherches doctorales (Goudi, 2009, 2011 ; Pignoli, 2017, 2018).

2 L'étude des taxonomies locales relève autant de l'anthropologie que de l'étude du langage, qui apparaît dès lors comme un angle d'approche permettant de périodiser les croyances selon deux points : l'organisation des êtres vivants entre eux et par rapport à l'homme, suivant les motifs utilisés pour les désigner. Les taxonomies actuelles, ni même celle en usage au siècle dernier ne peuvent représenter selon nous la succession des ontologies telles qu'elles sont recherchées par Alinei. Ce sont bien les études motivationnelles, révélant des types d'associations selon les signifiants qui, répétées, peuvent déboucher sur la mise en valeur de principes invariants. Si ce travail a été effectué à partir de corpus régionaux, aucune étude n'avait encore porté son attention sur la diatopique des classes motivationnelles et de surcroit, en termes de fréquence. Dans la phytonymie arbëreshë, $60 \%$ des noms sont formés selon un phytomorphisme, $16 \%$ selon un anthropomorphisme et la dernière part semble être distribuée entre les zoomorphismes et les motivations incertaines. La multiplication de ces études permet la comparaison des corpus afin d'identifier des tendances. C'est ce qu'Alinei proposait d'appeler des isomotifs, non plus établis sur la base d'une seule notion, mais sur un groupe d'êtres vivants. Nous proposons d'appliquer cette méthode pour analyser les noms d'algues en Basse-Bretagne et en Irlande. 
Tableau 1. - Définition des classes motivationnelles à partir de corpus régionaux.

\begin{tabular}{|l|l|l|}
\hline $\begin{array}{l}\text { Zoonymie à Lesbos } \\
\text { (Goudi, 2011) }\end{array}$ & $\begin{array}{l}\text { Phytonymie en Arbëria } \\
\text { (Pignoli, 2017) }\end{array}$ & $\begin{array}{l}\text { Entomonymie } \\
\text { en scandinave } \\
\text { (Bartoleschi, 2020) }\end{array}$ \\
\hline $\begin{array}{l}\text { Motivations descriptives "plus } \\
\text { importante» }\end{array}$ & $\begin{array}{l}\text { Motivations } \\
\text { phytomorphiques 60\% }\end{array}$ & Objets \\
\hline Zoomorphismes & $\begin{array}{l}\text { Motivations } \\
\text { Zoomorphiques 16\% }\end{array}$ & Animaux \\
\hline Anthropomorphismes & Anthropomorphismes & Étres humains, créatures \\
\hline Motivations opaques & Motivations opaques & - \\
\hline Motivations incertaines & Motivations incertaines & - \\
\hline
\end{tabular}

\subsection{Les sources disponibles en phyconymie dialectale}

3 Les phyconymes sont présents dans de nombreux ouvrages spécialisés. En dialectologie d'abord, avec les enquêtes de terrain effectuées par Le Berre (1968) en Basse-Bretagne, par Ropars dans le cadre de l'ALCAM², puis en Irlande, dans la réponse aux questions 1085 à 1088 du Linguistic Atlas and Survey of Irish Dialects (Wagner \& Ó Baoill, 1958). À partir des monographies locales, nous avons compilé les phyconymes présents à la fin des ouvrages dans un tableau. Les publications naturalistes contiennent aussi des noms vernaculaires qui ont été ajoutés lorsque la localité était explicitée par l'auteur. Des enquêtes de terrain réalisées en Irlande en 2019 puis en juillet 2021 en Basse-Bretagne ont permis d'affiner les étymologies, en demandant parfois le sens pressenti par l'informateur ${ }^{3}$. En compilant les données et en réalisant des enquêtes de terrain, nous avons pu réunir 622 phyconymes en Irlande et 1080 en Basse-Bretagne. Les attestations se trouvent en plus grand nombre dans les ouvrages de biologie (Brennan, 1950) et dans certaines monographies locales (Mac an Iomaire, 2000). Suivant les sources, l'association de la désignation dialectale et de l'espèce est plus ou moins fiable. Le nom commun est lui-même variant dans l'espace et implique d'établir une liste des équivalences. La remarque générale est que l'association espèce/désignation locale est plus précise dans le cadre d'une étude naturaliste, et la taxonomie populaire, plus détaillée dans le cadre d'une étude ethnolinguistique. Si l'association avec le nom scientifique est rarement précisée, nous avons associé lorsqu'il était possible certaines désignations à des espèces bien connues (ex : duilleasc « red weed»-Palmaria palmata). La diversité des phyconymes dépend principalement de l'usage local, de l'environnement et des sources.

4 Dans la majorité des cas, les êtres vivants nommés sont ceux qui ont une utilité pour les personnes qui les désignent, qu'elle soit révolue ou actuelle. Elles s'organisent ensuite selon une classification différente de celle utilisée par les biologistes bien que sur certains points elle s'en rapproche. Si le nombre d'espèces reconnues n'est pas total, la 
taxonomie populaire permet la multiplicité des désignations par espèce et la dissociation. C'est donc dans ce cadre que l'on trouvera des structures différentes, comme la saisonnalité, le type de récolte, la zone de récolte ou l'indication d'un emploi alimentaire de l'algue par les animaux. La systématique scientifique propose un pré-classement des algues en fonction de leur couleur : brune, verte ou rouge. Elle se ramifie ensuite sur la base de critères morphologiques : en lames, en spirales, dentées, à vésicules. À la différence de la systématique scientifique, la taxonomie populaire prend en compte une diversité de déterminants qui relève de leur provenance ou de leur mode de récolte.

\section{Méthode}

Dans un ordre donné parmi les êtres vivants (poissons, algues, coquillages), on évalue la proportion de signifiants par classe motivationnelle exprimée en pourcentage selon la méthode mise en place pour l'étude des phytonymes arbëreshë (Pignoli, 2018). Ensuite, on analyse la variation statistique par classe dans l'espace. Cette méthode permettrait peut-être, en multipliant les analyses et en étendant la zone d'étude, de mettre en valeur des zones de contact perceptuelles. Le biais principal prévu concerne l'impact statistique de l'incertitude concernant les motifs et leur opacité. Le deuxième concerne le nombre important de désignations prises en compte qu'il est plus difficile de réduire. Deux solutions s'offrent alors : 1) réduire le cadre géographique ou 2) réduire le champ sémantique d'analyse (Animaux - Oiseaux - Charadriiformes). Afin de contrôler les données, nous allons d'abord comparer les classes motivationnelles identifiées en Arbëria et à Lesbos et dégager les classes motivationnelles supplémentaires au niveau local. Dans un deuxième temps, nous allons donner des pistes de réflexion sur la variation motivationnelle statistique en Irlande et en Bretagne.

\subsection{Un exemple d'analyse motivationnelle à partir des phyconymes de la commune de Landéda, Finistère ${ }^{4}$}

6 Cette liste présente l'ensemble des désignations d'algues en usage dans la commune de Landéda. Elle est structurée de la manière suivante :

7 orthographe phonologisante [désignation en Alphabet Phonétique International]: Source/Initiales du locuteur. Définition en français. Nom scientifique en latin de l'espèce désignée. Témoignage éventuel de l'informateur pouvant aider à la compréhension générale du motif. Le motif en lui-même, c'est-à-dire l'interprétation de la valeur sémantique du signifiant que nous proposons. La classe motivationnelle à laquelle appartient la désignation.

- bijin [bi'3in] : désignation collective des algues à Landéda. Algae spp. Le motif est incertain. En considérant un ensemble de variantes, comme celles de l'île de Sein bedin et du sud-est bein on peut suggérer la présence à date ancienne d'une spirante interdentale [ð] présente également en gallois. La première hypothèse que nous proposons consiste donc à la comparer au gallois peithin «lanière, tuile $»^{5}$ (GPC online, 2020). La deuxième hypothèse proposée serait une variante d'un autre terme gallois ethin qui veut dire «broussaille » (Henry, 1900 ; Favereau, 2016). MOTIVATION : la motivation se ferait selon un transfert phytonymique, passant de la désignation d'une terrestre à une plante marine, un réflexe regroupé dans la classe motivationnelle des phytomorphismes. 
- bijin bleuñ [bi Zin blœ], ALB : [ar blõ], Pd: longues laminaires. Laminaria hyperborea. Une confusion est possible avec Laminaria digitata. MOTIVATION: bijin «algues »+ bleuñ «fleurs » = « algues aux fleurs ». Ce motif est probablement dû aux épiphytes, ces algues qui utilisent d'autres algues comme support pour se développer, il est également présent dans le gaélique scothach (Mac an Iomaire, 2000). Cette motivation est descriptive.

- bijin du [bi'zin dy] : VC, YLG \& YK, JP : [be'zin dy], coll. : le goémon noir, fucus ou fucales, que l'on voit accrochées aux rochers en haut d'estran. Fucus spp. Bijin générique incertain " goémon, algue » + $d u$ " noir » = " goémon noir ». " Distaget e vez gand an dourn pe gand eur fals vien. Tud ar fermioù bras a deue da gerhet bijin du hag a zigase keuneut. Trafik a ioa» (YK) [«On le détache à la main ou avec une faucille. Les gens des grandes fermes venaient chercher du goémon noir à la côte et amenaient du bois. Il y avait du troc. »].

- bijin du bien [bi'zin dy bi:en], ALB coll. : algue brune. Pelvetia canaliculata. Bijin «algues »+ $\mathrm{du}$ «noir»+ bien « petit» = « petit goémon noir ». MOTIVATION : la motivation est descriptive.

- bijin kalpan [bizin 'ka:lpãn]: JP \& VC, YLG: [kalpãn], ALB coll. : fucus dentelé. Fucus serratus. Algue préférée pour nourrir la terre car elle ne possède pas de vésicules contrairement à A. nodosum, F. vesiculosus et F. ceranoides. Bijin « algue » + kalpan « motelle, loche de mer, Gaidropsarus vulgaris » = « algue à motelles ». MOTIVATION : la motivation est descriptive.

- bijin reun [bi Zin 'rœ:n], ALB : pas de désignation commune en français. Cystoseira baccata, br. reun "soie», probablement de par sa morphologie générale. D'autre désignations d'algues sont motivées par les poils d'animaux : br. lostou kezeg, -lern « queues de chevaux, renards ». MOTIVATION : la motivation est descriptive.

- bijin ston [bi'zin 'ston], ALB: laminaire. Laminaria digitata. Confusion possible avec L. hyperborea. tonn « qui se décroche du fait du reflux et des vagues pour se déposer sur le bord », « goémon d'épave » serait la traduction plus proche. MOTIVATION : la motivation est descriptive.

- butun [bytyn], ALB : désignation collective pour l'Alaria esculenta. Butun veut dire «tabac ». MOTIVATION : c'est une désignation descriptive puisqu'elle se base sur l'aspect du tabac.

- bijin torr [bi'zin 'to'r], Pd: goémon d'épave. Laminaria hyperborea, principalement. «Ar bleuñ hag ar bijin torr vehe ramasset gand er rachtell»(YK) [« Le bleuñ et le bijin torr se ramassaient au râteau »]. Torr « cassé, détaché » = " goémon d'épave ». Le gaélique múr briste est formé selon la même motivation. MOTIVATION : c'est une désignation descriptive.

- glandour (-glaz) [glãndur], ALB : [-glaz], JP : [-gwer], MG : désignation collective pour les algues vertes en général. Ulva lactuca mais aussi parfois les enteromorphes Enteromorpha sp. La laitue de mer est dite plus particulièrement saladenn [sa'la:den] « salade ». Témoignage de VC en mai 1985 : «on en voit de plus en plus ». En français local on parle du glandour [lø 'glã'dur] ms. C'est l'algue qui se dépose sur les poches à huîtres, PT. glan « laine, gluant » + glaz " verdure » = « laine ou gluant d'eau + de verdure », probablement en raison des filaments et de l'aspect visqueux d'Enteromorpha sp. La distinction avec les algues d'eau douce qui se forment dans les eaux stagnantes, étangs et les lavoirs est faite en précisant sa couleur: glaz/gwer. MOTIVATION : la motivation est descriptive.

- grahetach [gra'hetaf], JP : [-3], ALB coll. : /+u/ pl., YLG, PAB64 : laminaire sucrée, large et ondulée. Saccharina latissima. Elle peut être confondue avec Alaria esculenta selon A. Le Berre. Grahed "vieilles (poisson)» + suff. -ach « matière à ", sur la base du biotope supposé des vieilles. MOTIVATION : la motivation est descriptive et renvoie à l'habitat d'une autre espèce. 
- kinvi ['ki:nvi], JP \& YLG : fucus denté. Fucus ceranoides. kinvi «mousse végétale ». Motivation descriptive effectuée selon un phytomorphisme.

- kore, korre ['ko re], YLG \& JP : ['korre], ALB coll. : longues algues en filaments. Chorda filum et Himanthalia elongata. En français local, haricot d'mer [ari'ko dmer] : PAP. Voir. alt. lañsou. Le motif est incertain, on peut noter le galicien correa de même sens (Ríos Panisse, 1983) et le gaelique corrleach « H. elongata » à Rinvyle (Colgan, 1911). Le terme est probablement motivé par le nom de la courroie br. koreenn lat. CŎRRIGGIA (FEW : VII). MOTIVATION : il s'agirait donc d'une motivation descriptive.

- lañsou [lãsu], JP, YLG : longues algues en filaments. Chorda filum peut-être aussi Himanthalia elongata. En français local, haricot d'mer [ari'ko dmer] : PAP. lañsou «lances»= «algues élancées ». MOTIVATION : la motivation est descriptive.

- lostou kezeg [lo'stu 'ke:zEk], YLG \& JP : cladophores des rochers, Cladophora rupestris. «Enebour d'ar pioka » (JP) [ « il fait concurrence au Chondrus cripus»]. Nul selon YLG du fait qu'il n'y ait pas beaucoup d'iode dedans. MOTIVATION : « queues de chevaux », en raison de la morphologie générale de l'algue. Phytomorphisme.

- meles (-rouz) ['me:les] [-k ?], JP : [-t], YLG : [-'ru:z], ALB coll. : zostères marines. Zostera marina ou herbier par extension. MOTIVATION : "doux, agréable, sucré », gallois gwymon melys de même sens, cfr. gael. milis « doux, sucré », br. rouz « rousse, brune ». On l'utilisait une fois sèche pour rembourrer les matelas.

- mosper(-n) ['mo'sper] : JP, VC : ['mo'spern], YLG coll.: Codium spp., ['mor spern], ALB : Bifurcaria bifurcata. Plus fréquent en Rade de Brest, mor «mer » + spern "aubépine »= "aubépine de mer». MOTIVATION: la motivation est descriptive et réalisée selon un phytomorphisme.

- pioka ['pjo'ka] : JP, YLG \& YK, parfois [ta'pjo'ka] : MG, AmR ou [ta'bjo'ka], MG : Carragheen. Chondrus crispus principalement mais parfois aussi Mastocarpus stellatus. En français local [tapjoka] : PAP. Il s'agit d'un emprunt récent issu de la remotivation du terme tupi tipi'óka d'origine incertaine passé en portugais depuis le Brésil puis en fr. tapioca, agent gélifiant présent dans les racines du manioc que l'on retrouve également dans le carragheen, le carraghénane, code E407 ou agar-agar. «Ar pioka vez distaget dah ar herreg. Deomp da glask an tabioka gad ar boutog. Ar re-ze vez lakeat var an teñvenn, cheñchet tu deho, da veza skañv ha gwerzhet da houde » (YK) [« On détache le pioka des rochers. Allons chercher du pioka avec un panier. On les met sur la dune, on leur change de côté pour qu'ils soient légers, puis on les vend »]. MOTIVATION : la désignation est descriptive.

- pioka du [pioka 'dy] : ALB. Désigne l'algue mouillée, elle devient rose après avoir été rincée par la pluie puis blanche une fois sèche. Chondrus crispus. Pioka «Chondrus crispus»+ du « noir » = « pioka noir » ou « Chondrus noir ».

- pour ['pu:r] : JP \& YLG, ALB coll. : Fucus noduleux. Ascophyllum nodosum. fr. local : l'asco [la:sko] : MG. MOTIVATION : le motif est incertain, la morphologie ne semble pas a priori en constituer le motif.

- radenn vehin [raden 'vehin] : ALB. Fucus vesiculosus. radenn «fougère " + bezin " goémonfougère». MOTIVATION : la motivation est descriptive et se fait selon un transfert phytonymique effectué sur la base de la morphologie. Cette désignation a probablement motivé le toponyme nautique Radenog [ra'de:nok] d'après YK.

- radenn(-mor) [ra:den] : JP, VC: [-mo:r], YLG coll.: fucus spiralé, Fucus spiralis. radenn « fougère sf. » + mor « mer » = « fougère (-de mer) », transfert phytonomique sur la base de la morphologie. MOTIVATION : la motivation est descriptive et phytomorphique.

- saladenn [sa'la:den] sing. : VC, YK : Ulva lactuca, la laitue de mer. Emprunt au fr. salade+ désinence -enn, marque du singulatif, nom de la laitue cultivée en breton saladenn appliqué à 
l'algue en raison de sa morphologie et de sa couleur. MOTIVATION : la motivation est donc descriptive et phytomorphique.

- sier dour [sier 'du:r], ALB : Fucus spiralis, sier « sacs, poches » + dour « eau » = « sacs, poches d'eau ", en raison des vésicules probablement. MOTIVATION : la motivation est descriptive.

- tali ['ta:li], coll. : [en da'li:en] sg., ALB : les laminaires en général. Laminaria spp. Le terme est probablement emprunté au lat. thallus, gr. $\theta \alpha \lambda \lambda_{0}$ ' $\varsigma$ " jeune pousse, jeune branche », par la biologie puis l'industrie. Cet emprunt est probablement récent et date de la période du développement de l'industrie de l'iode sur les côtes du nord Finistère à la fin du XIX siècle.

- tali leaz [ta:li 'le'az], ALB : laminaire jaune. Laminaria ochroleuca. Le motif est incertain leaz « lait » = « laminaire de lait, laiteuse », de par sa couleur probablement.

- tali moan [tali 'mo:ãn] : JP \& YLG. Laminaria digitata. MOTIVATION : "goémon mince ». Elles sont brulées dans des fours pour en extraire la soude.

- tali penn [tali 'pe'n] : JP, YLG, coll. : les grandes laminaires du fond qui se détachent en mai après la marée d'équinoxe et qui se coupent à la guillotine. Laminaria hyperborea. Elles étaient brulées dans des fours en pierre creusés dans la dune, pour en extraire la soude.

- toser ['to'scr], JP : ['to:scr], YLG : [toser], ALB coll. : laminaire avec poche et dentelles à la base. Saccorhiza polyschides. La motivation est incertaine, le dictionnaire de Favereau propose «lanières de cuir, baudrier» pour toser. MOTIVATION : on peut alors entrevoir une motivation descriptive formée sur l'analogie avec le cuir. Dans cette direction, on peut aussi ajouter la motivation de korre.

- pioka deliou karotes ['pjo'ka deKu karo:tes], JP : « sorte d'algue rouge », pioka « Chondrus crispus »+ deliou «feuilles » + karotes «carottes». "Chondrus crispus aux feuilles de carottes». MOTIVATION : la motivation est descriptive, basée sur phytomorphisme puisqu'elle met en relation la forme commune des feuilles de carottes avec celle de l'algue.

- telles [teLEs], JP : [-z], ALB coll. : la dulse. Palmaria palmata, surtout. La motivation est incertaine. Une hypothèse, établie à partir des variantes gaéliques propose un composé de duille « feuille » et uisce « eau » (Jamieson, 1808 ; MacBain, 1911 ; Vendryès \& Lambert, 1996). La première séquence en breton est probable br. delienn « feuille » mais impossible dans la deuxième dour « eau ». Elle rentre donc dans la classe des motivations incertaines.

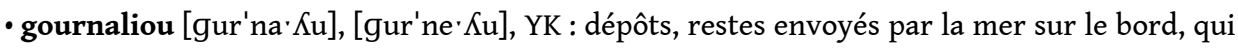
contiennent principalement du goémon. «pa vez mare, e teu gand ar mor var ar bord, bejin, restachou toud hag e veze ramasset toud an dra-he : an dra-he ar gourneliou » (YK) [" à la marée, il vient sur le bord des algues, des rebuts de toutes sortes et on ramassait tout ça : c'est ça les gournaliou »]. MOTIVATION : forme locale de gourlen, avec métathèse + suff.pl. -ioù : gour/ bour/mour « mer »+ leun « un/du plein » = « plein(s) de mer ».

\section{Des concordances lexicales observables à l'échelle Manche-Atlantique}

8 Nous pouvons observer deux concordances principales dans l'aire celtique. La première concerne la désignation des algues en général établie sur la base d'une alternance FW-/ GW- (carte a) fr. oïl goémon, goamon br. Goumon, corn. Gumman, gall. Gwymon, gael. feamainn, feamnach. Remarquons qu'une concordance existe aussi dans l'aire germanique fr.oïl vra, varech ang.dial. wrack « rebus, épave ». La deuxième concerne la désignation de la dulse (carte $b$ ) une algue rouge dont on sait qu'elle était consommée en Irlande et en Ecosse et dès le Néolithique par des caprinés dans les Orcades (Balasse, Mainland \& Richards, 2009). Si l'étymologie proposée par Vendryès fonctionne pour le 
gaélique : duille "feuille + uisce " eau », l'équivalent en breton et en gallois reste incertain, le terme pour l'eau étant dour. La désignation de la zostère présente également une concordance établie à partir d'un dérivé du nom du miel gaul. *meli $(t)$ "doux, agréable » et minio-, meno- «doux» (Delamarre, $2008: 224,227):$ br. melez, melest, gael.irl. miliunach, meilscéanach, gael.ecc. milearach, bilearach, minearach (Zostera marina). Si l'emploi de la plante pour le rembourrage des matelas est largement attesté, sa consommation par l'homme l'est beaucoup moins. On fera donc référence au ressenti du naturaliste norvégien Perh Kalm (1716-1779) : «Kalm, ever curious, tried its taste, and found it agreeable: 'when one chews it, it tastes sweet and pleasant' ${ }^{6}$ (Alm, $2003: 641$ ). D'autres rapprochements restent envisageables comme le br. korle (Dossen) et le gael. coirleach (Chorda filum et Himanthalia elongata), tandis que d'autres semblent plus récents br. saladenn et gael. saladaí (Ulva spp.).

Figure 1. - a. Désignation des algues en général ; b. Désignation de la dulse ( \pm Palmaria palmata).

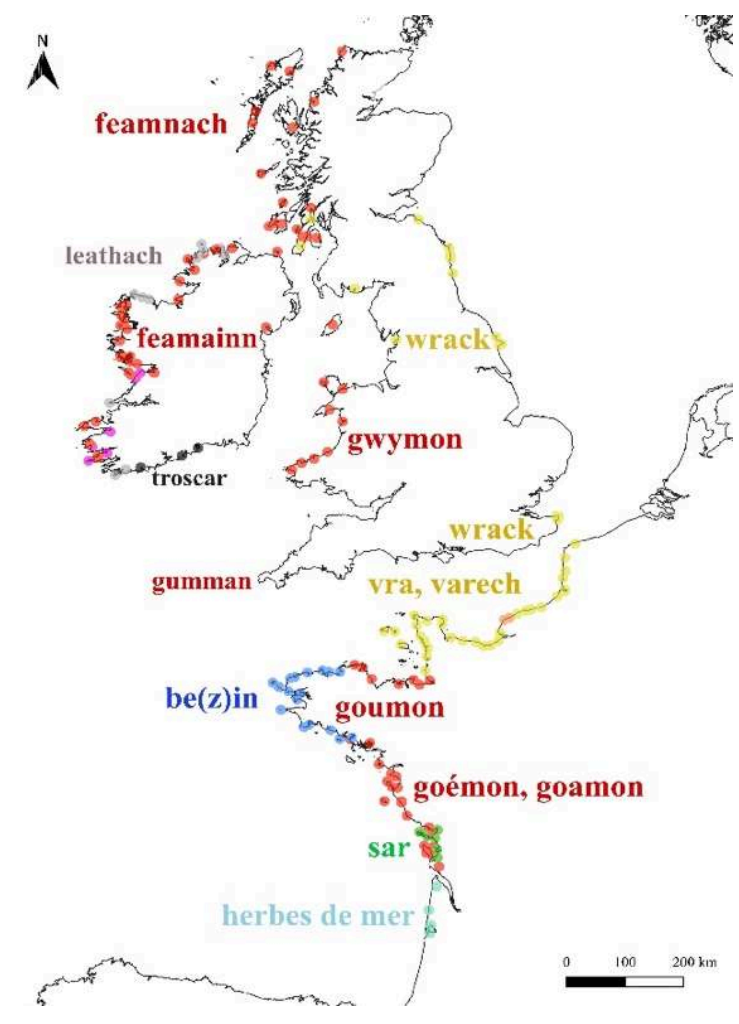




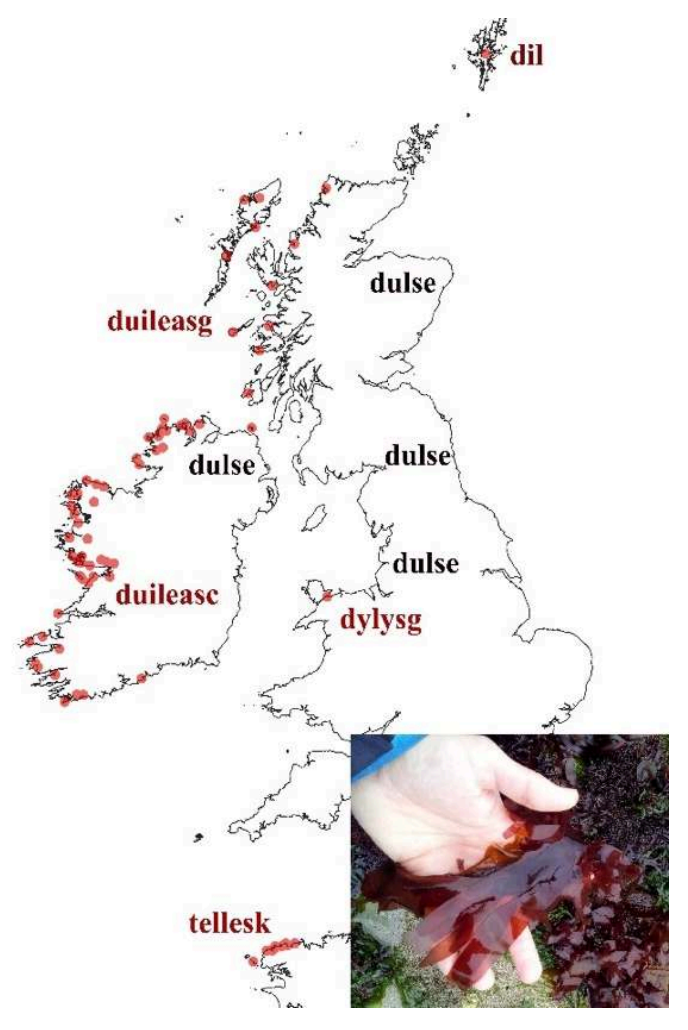

\section{Classes motivationnelles}

\subsection{Motivations descriptives}

\subsubsection{Structures avec un terme générique}

Les goémons d'épave qui s'amassent à chaque marée le long de la côte et en nombre plus importants aux marées d'équinoxe sont principalement constitués de laminaires. C'est la seule classe englobante qui semble exister parmi les algues en Irlande outre les structures établies sur un nom de couleur. En Basse-Bretagne, on trouvera ensuite le goémon de coupe ou de rive bezin trouc'h, bezin du, opposé par son milieu au goémon d'épave bezin torr ou bezin peñse, gael. Múr briste "goémon cassé, d'épave». Des différences seront faites notamment suivant la saison de récolte pour une même espèce par la modification du spécifique (ex: múrach bealtaine en mai et múrach foghmhair en automne "L. hyperborea »). Les génériques sont utilisés pour désigner des espèces autrement considérées indépendantes dans la nomenclature comme faisant partie de la même "sous-famille ». À partir du br. tali (Laminaria sp.) on distingue alors le tali ebrel "laminaire d'avril» ou tali penn "laminaire de tête" (L.hyperborea), tali moan (L. digitata) «maigre laminaire " et tali dantelez « laminaire à dentelles " (Saccharina latissima). En gaélique aussi, la désignation des algues en général sert de générique : feamainn: feamainn mhilis "goémon sucré, doux" (Saccharina latissima), feamainn bhuilgíneach "goémon à vésicules" (Fucus vesiculosus), gael. bolg "vésicule, vessie, estomac ». Les génériques concernent principalement les laminaires et les fucus, les deux genres qui servent à faire de la soude et de l'engrais. La focale est plus précise au fur et à mesure que l'on se rapproche du milieu dans lequel elles évoluent. Comme pour les oiseaux, si l'espèce est distinguée par les gens de côte, dans l'intérieur des terres, on 
va englober les espèces par un terme générique, avec parfois, une précision du milieu br. laboused mor « oiseaux de mer ».

\subsubsection{Structures avec un nom de couleurs}

On retrouve les couleurs qui ont servies à établir la nomenclature scientifique : vert (Chlorophyta), brun (Phaeophyceae) et rouge (Rhodophyta). Elles permettent de se représenter un ensemble mais intrinsèquement, à déterminer un type de récolte br. Bezin $d u$ "goémon noir ", appliqué à toutes les fucales (brun-noir) qui sont récoltées avec une faucille ou à la main. En Irlande, une seule attestation montre que l'idée de famille peut être établie sur la base de la couleur : clann dearg « famille rouge » dont fait partie la Polysiphonia fastigiata ${ }^{7}$. Les couleurs arrivent généralement dans la seconde partie de la combinaison:-glas, -dubh, -donn, -buí, -dearg et rarement dans la première : gael. glasán, br. glasvez "Ulva sp. 'verdure' ». Les algues rouges changent généralement de couleur lorsqu'elles sont détachées et exposées à la pluie, on retrouvera alors une distinction selon l'aspect : likenn zu «-noir ", liken venn «-blanc » (à l'île de Batz pour Chondrus crispus).

\subsubsection{Structures avec un nom d'objet}

Les structures établies à partir d'un nom d'objet révèlent souvent la morphologie générale de l'algue : br. lasou " lacets ", rubanou/seizennou " rubans », spanell « spatule ». On renvoie simplement la forme des objets de la vie quotidienne sur les individus. La désignation du Chorda filum et d'Himanthalia elongata br. korre est probablement motivée par le nom de la courroie.

Figure 2. - Désignation du Chorda filum (côte nord-Finistère).

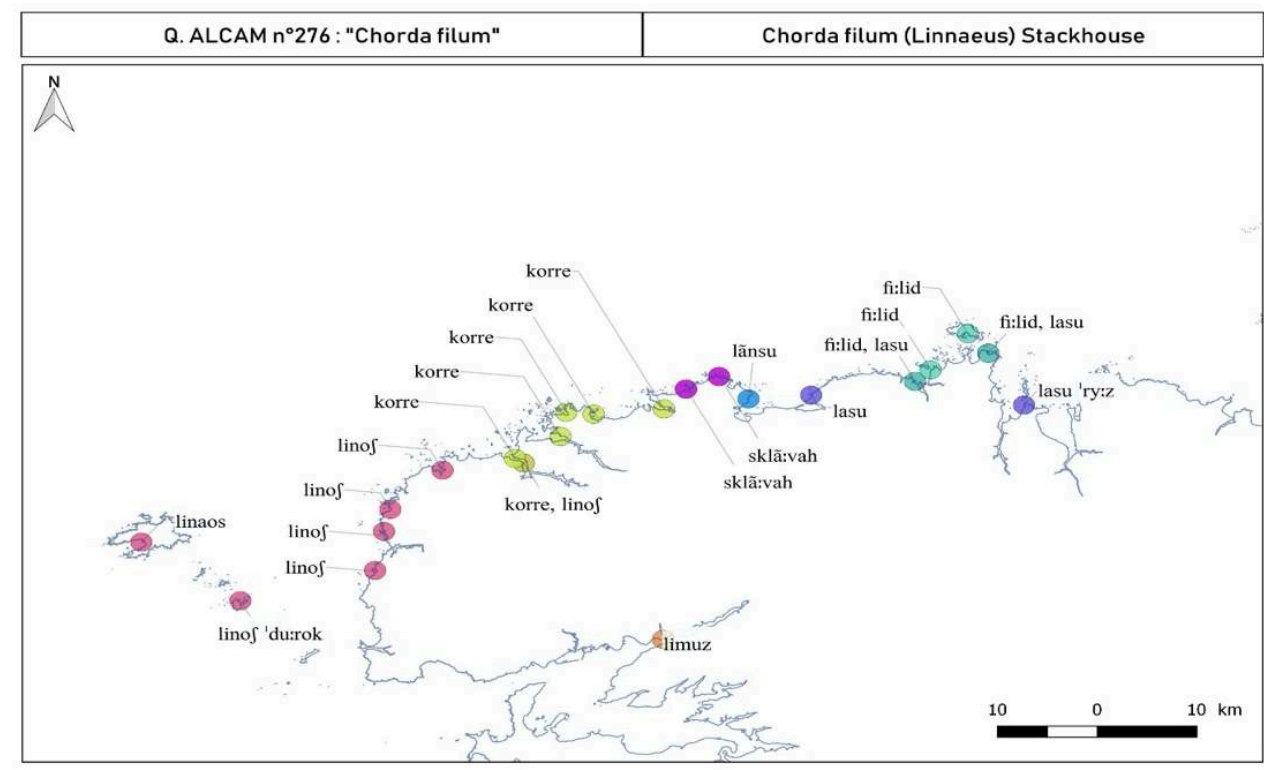

\subsubsection{Phytomorphisme et transfert terre-mer}

Les transferts d'images se font uniquement dans un sens: on applique l'image d'un phytonyme terrestre à une réalité marine. Les exemples les plus transparents sont les 
combinaisons formées à partir du br. mor «mer» : mospern (Litophyllum spp. / Codium tomentosum) et spern-mor (Cystoseira baccata) « aubépine de mer ». Des distinctions entre espèces terrestres peuvent aussi faire l'objet d'un transfert radenn (Alaria esculenta) «fougère » et radenn puñs (Halidrys siliquosa) «scolopendre ». L'espèce de référence peut être cultivée : poix, fève, carotte, salade, choux, figue, poireau, lin, prunelles ou non : bruyère, fougère, aubépine, chêne, jonc. Ce transfert unilatéral est plus important en Basse-Bretagne qu'en Irlande ou un seul exemple existe : copóg (L. digitata), copógach woher (S. latissima) « rumex ».

\subsubsection{Le son et l'onomatopée}

13 La seule onomatopée concerne la désignation des fucus à vésicules, qui lorsque l'on appuie dessus produisent un petit claquement. Le mode de formation lexicale est assez proche de l'arbëreshe puisque l'on retrouve le gabarit trilitère TR.K, à la différence, que ce son est accompagné d'un suffixe nominal pluriel masculin ou au féminin: br. strakerien-/strak-/ «éclat »+ /-erjen/ ou br. bezin/strak-/ /-e're:zed/ «F. vesiculosus » désignant généralement des personnes ou des machines.

\subsubsection{Habitat de l'algue}

Cette catégorie descriptive concerne le biotope des algues et plus particulièrement leur support : gael. carraigín « rocher + dim. » (Chrondrus crispus), br. lehid « vase » (Zostera marina) ou leur position sur l'estran br. Bezin darze "goémon de basse mer " Fucus serratus. La zone de récolte, qui est intimement liée à l'habitat, est aussi présente dans certaines désignations br. pioka heleyer « pioka des roches les plus éloignées ».

Les désignations de certaines algues indiquent aussi la présence d'animaux marins br. Bezin steredennou (Codium tomentosum) « goémon à étoiles de mer ", bein silio (Saccharina latissima) « congres », bijin du kalvan (Fucus spp.) «-noir à motelles », bijin garvet (Fucus spiralis) "gravettes», gael. leaba portáin (Furcellaria spp.) «lit de crabe». Ces informations contenues dans les désignations sont issues d'une observation fine et prolongée de l'environnement.

\subsubsection{Emploi de l'algue}

Les désignations dialectales donnent également à voir l'utilisation de l'algue, qu'elle soit révolue ou actuelle : br. Bijin yod, bejin farda yod (Chrondrus crispus) « (pour préparer) la crème » désigne une algue qui contient une substance gélifiante utilisée localement pour la préparation du flan. La zostère marine br. gwelaj (Zostera marina) «literie» servait à rembourrer les matelas et comme évoqué plus haut, elle a probablement été consommée br. melez, gael. miliunach « doux, agréable». À Lisduff (Co. Offaly) dans les années trente, on rapporte que les algues étaient mangées avec des pomme-de-terres. L'informateur d'une soixantaine d'année à l'époque, explique que c'est une pratique qui a disparu depuis longtemps ${ }^{8}$. Le bezin saout (P. palmata) «à vaches» indique au moins que l'algue était consommée par les bovins ${ }^{9}$ et le gael. múirín na muc par les porcs. Notons que ces désignations sont en usage principalement dans les îles (île de Batz, Valentia Island). Le br. Bejin tan, bezin forn (Fucus spiralis) montre que cette espèce était utilisée pour la combustion, à défaut de bois. 


\subsection{Motivations zoomorphiques}

\subsubsection{Zoonymes}

17 La présence du motif "cheval» en Basse-Bretagne semble noter la sexualité et l'attribut mâle en particulier : br. marh, pl. marhou (Saccorhiza polyschides) qui désigne une laminaire avec un bulbe à la base du stipe. Cette lecture, pressentie par Le Berre (1973:494) en zoonymie marine, montrait que les mâles étaient motivés par les allosèmes «taureau ", «cheval », «bouc » et " verrat ». L'association de ces animaux semble fonctionner dans l'ensemble vivant : targazh (tar-- "taureau » + kazh « chat ») en Basse-Bretagne et en Ecosse : gael. tarbh-shiolag (vive, considérée comme le mâle du lançon). On retrouve aussi le chien pour désigner des laminaires: gael. madra, br. Penn ki.

\subsubsection{Parties du corps de l'animal}

Les structures «partie du corps animal + animal» remarquées en arbëreshe (Pignoli, 2018) sont également les plus fréquentes dans phyconymie celtique. Elles concernent uniquement deux parties du corps : la queue : br. Lostou louarn (Furcellaria lumbricalis) "queues de renard", lostou kezek (Cladophora rupestris) "queues de chevaux ", gael. eireaball cait (Incertae sedis) « queue de chat ", la patte et la griffe : gael. cos sionnaigh (Pelvetia canaliculata) "patte de renard»; br. Pioka patou yer (C.crispus) « pioka à pattes de poules ${ }^{10}$ ", gael. crúba préacháin (Halidrys siliquosa) en référence à la forme des lobules. Le renard et le cheval sont les animaux dont les parties du corps sont les plus fréquemment utilisées pour motiver les noms d'algues. L'animal de référence n'est pas toujours donné en gaélique: cosa cruadha (F. vesiculosus) "pattes dures", cosdhubh "patte noire», sraoilleach (Alaria esculenta) «à queue», caisíneach (P. canaliculata) «à petites pattes». Une analyse des motifs zoomorphiques dans la phytonymie de Basse-Bretagne compilée à partir de dictionnaires montre qu'une proportion importante des désignations sont motivées par le motif « chien» :

Les motifs zoomorphiques dans l'Herbarum vernaculi (Duros, 1991).

\begin{tabular}{|l|l|}
\hline Motif zoomorphique & $\begin{array}{l}\text { Nombre d'espèces } \\
\text { concernées n = 171 }\end{array}$ \\
\hline Chien & 18 \\
\hline Lièvre & 13 \\
\hline Loup & 10 \\
\hline Coucou & 9 \\
\hline Souris, porc, oiseau & 8 \\
\hline Cheval, serpent, oie, verrat, bouc & 7 \\
\hline Chat & 6 \\
\hline
\end{tabular}




\begin{tabular}{|l|l|}
\hline Renard, poule & 5 \\
\hline Corbeau, crapaud, bœuf, vache, poux & 4 \\
\hline Âne, chèvre & 3 \\
\hline Insecte, coq & 2 \\
\hline $\begin{array}{l}\text { Ours, dauphin, veau, chat, oiseaux de mer, alouette, grive, bélier, } \\
\text { canard, geai, varon, rat, abeille, vipère, mouton, poulain. }\end{array}$ & 1 \\
\hline
\end{tabular}

\subsection{Motivations anthropomorphiques}

\subsubsection{Relations parentélaires}

Une relation parentélaire entre les algues n'a été observé que par deux fois en Irlande : mathair an chreathnaí "mère du Palmaria palmata" (Lucas, 1986) à Ros Goill et máthair an duillisc (Osmundea pinnatifida) dans le sud du Connemara (Brennan, 1950). Ces désignations montrent une relation de parenté entre les individus d'une même espèce, la mère portant des coquillages et les autres non. Parmi les noms d'animaux marins, seule la patelle br. tadeu "pères » et probablement le tacaud br. tad-kozh (Trisopterus luscus) « grand-père » semblent concernés par une désignation parentélaire directe.

Dans la majorité des cas, l'évocation de la parenté est indirecte: la grand-mère; spillou mamm goz «les épingles de grand-mère" ( Scandix pecten veneris, Erodium Cicutarium \& Geranium robertianum), krampouz mamm goz « la crêpe de grand-mère " (Umbilicus rupestris), chapeled ar vammgoz "le chapelet de la grand-mère " (Arrhenatherum elatius, var. bulbosum), la mère; louzaouenn arvamm, louzaouenn ar mammou « la plante de(s) mère(s)» (Matricaria chamomilla), le chef de famille; skouarn an ozac'h koz "l'oreille du vieux chef de famille» pour l'achillée millefeuille (Achillea Millefolium) ou encore bara an ozac'h koz (Pulmonaria Angustifolia).

\subsubsection{Personnages magico-religieux et références à la religion chrétienne}

21 Il y a au moins une référence à un personnage magico-religieux gael. gruaig na caillí mara « cheveux de la vieille femme + de mer » traduit en anglais par « sea witch's hair " (Mac an Iomaire, 2000). Ce motif apparait régulièrement dans les désignations d'animaux : raie, loche, petite roussette, cormoran, grand tétras, chouette, cloporte, criquet, coquelicot, sans que nous puissions établir de dénominateur commun. Le diable est une référence fréquente en zoonymie et en phytonymie mais ne semble pas concerner les algues. En zoonymie marine, il semble associé à des animaux qui peuvent piquer avec du venin ou non. Seule Sainte-Barbe semble être évoquée pour les algues : plant santez barba (Codium sp.) à partir du générique plant pourtant réservé aux plantes terrestres. Les saints sont plus présents pour motiver les noms d'animaux marins sont Pierre, Jean, Jacques, Nicolas et Paul, ce qui montre une faible influence de la culture celtique insulaire sur l'hagiographie bretonne. Les désignations se rapportant à la tenue des prêtres sont considérées comme des anthropomorphismes bien qu'elles soient essentiellement descriptives br. lostou, bouchou beleien « queue, touffe de prêtre ». 
Les références à la religion chrétienne semblent plus prégnantes en Basse-Bretagne qu'en Irlande.

\section{Conclusion}

En science, le mode de formation lexicale pour les êtres vivants s'opère par la mise en valeur du spécifique dans une catégorie générique et la règle impose de motiver les désignations par un élément descriptif (la taille, la couleur, la forme). Ce processus semble également majoritaire dans la phyconymie dialectale. On l'observe à Lesbos en Grèce (Goudi, 2011 : 240) et en Arbëria (Pignoli, 2018 : § 18). Là où il diffère, c'est dans la diversité des critères, descriptifs et symboliques, reflétant des pratiques locales associées à ces êtres vivants sur le long terme. Dans la phyconymie de Landéda, 83,8 \% des noms impliquent des motivations descriptives dont $38,7 \%$ sont des phytomorphismes, 12,9\% impliquent une analogie avec des objets et 3,2 \% correspond à la valeur $\mathrm{du}$ seul zoomorphisme relevé «lostou kezeg». Il n'y a pas d'anthropomorphismes et $12,9 \%$ correspond à la proportion de motivations incertaines sur la base de 31 phyconymes. À titre de comparaison, la répartition des classes motivationnelles à Máinis (Co. Galway) est la suivante : 55,2\% descriptives, 7,8 \% de phytomorphismes, 5,2\% impliquent des objets, 23,6\% de zoomorphismes, 2,6\% d'anthropomorphismes "gruaig na caillí mara » et 18,4 \% de motivations incertaines sur 38 phyconymes. La poursuite de cette étude à partir du corpus zoonymique de l'Atlas linguistique des côtes de l'Atlantique et de la Manche permettra de détailler les classes motivationnelles récurrentes et de mieux comprendre leur fonction.

\section{BIBLIOGRAPHIE}

ALINEI Mario, 1983, « Arc-en-ciel », ALE I, 1, cartes 6-9, commentaire, p. 47-80.

ALINEI Mario, 1996-2000, Origini delle lingue d'Europa: La teoria della continuità, Bologna, Il Mulino.

ALm Torbjørn, 2003, « On the Uses of Zostera marina, Mainly in Norway », Economic Botany, ${ }^{\circ}$ 57, 4, p. 640-645.

BALASSE Marie, MAINLAND Ingrid, RICHARDS Michael P., 2009, « Stable isotope evidence for seasonal consumption of marine seaweed by modern and archaeological sheep in the Orkney archipelago (Scotland) », Environmental Archaeology, n 14, 1, p. 1-14.

BARTOLESCHI Claudio, 2020, « La motivazione lessicale dei nomi d'insetto in tedesco e nelle lingue scandinave », Géolinguistique [En ligne], 20 | 2020, mis en ligne le 01 décembre 2020, consulté le 13 octobre 2021. URL : <http://journals.openedition.org/geolinguistique/2032> ; DOI : <https:// doi.org/10.4000/geolinguistique.2032>.

BRENNAN Agnes T., 1950, Notes on some common Irish seaweeds, Published by the Stationery Office; To be puchased directly from the Govt. Pub. Sale Off., G.P.O., ([Catalogue lists], Ireland. Stationery Office). 
DALBERA Jean-Philippe, 2006, Des dialectes au langage. Une archéologie du sens, Paris, Honoré Champion éditeur.

DELAMARRE Xavier, 2008, Dictionnaire de la langue gauloise : Une approche linguistique du vieux-celtique continental, $3^{\mathrm{e}}$ édition, Paris, Errance.

DuRos Fanch, 1991, Herbarum vernaculi. Lexique du nom des plantes en breton, Moëlan-sur-Mer, La Digitale.

FAVEREAU Francis, 2016, Geriadur Bras/Grand Dictionnaire Français-Breton ; Breton-Français, (<https:// geriadurbrasfavereau.monsite-orange.fr>).

Goudi Maria, 2009, « Aperçu d'une étude motivationnelle de certains zoonymes dans les parlers de l'île de Lesbos (Grèce) ", Géolinguistique, nº 10, p. 71-91.

GOUDI Maria, 2011, Étude motivationnelle de la zoonymie dialectale dans les variétés linguistiques de l'île de Lesbos (Grèce), thèse de doctorat, université de Grenoble.

GPC online, 2020, Geiriadur Prifysgol Cymru, University of Wales, Centre for Advanced Welsh and Celtic Studies.

GUIRAUD Pierre, 1967, Structures étymologiques du lexique français, Paris, Larousse.

HENRY Victor, 1900, Lexique étymologique des termes les plus usuels du breton moderne, Rennes, J. Plihon \& L. Hervé.

JAMIESON John, 1808, An Etymological Dictionary of the Scottish Language, Edinburgh, University Press.

LE BERRE Alain, 1968, «Influence des noms de goémons en breton sur la toponymie nautique des côtes du Léon ", Études Celtiques, nº XII, p. 202-235.

LE BERRE Alain, 1973, Ichthyonymie bretonne, thèse de doctorat, Brest, université de Brest.

LuCAs Leslie W., 1986, Cnuasach focal as Ros Goill, Baile Átha Cliath, Acadamh Ríoga na hÉireann (Deascán foclóireachta).

MAC an IOMAIRE Séamas, 2000, The shores of Connemara, Kinvara, Co. Galway, Tír Eolas.

MACBAIN Alexander, 1911, An Etymological Dictionary of the Gaelic Language, Stirling, Eneas MacKay, Glasgow.

MARKUS Manfred, 2021, English Dialect Dictionary Online: A New Departure in English Dialectology, Cambridge, Cambridge University Press.

PIGNoli Maria Luisa, 2017, Les désignations des plantes sauvages dans les variétés arbëreshe (albanais d'Italie) : étude sémantique et motivationnelle, thèse de doctorat, université Côte d'Azur (ComUE).

PIGNoli Maria Luisa, 2018, « Une étude de la motivation sémantique en domaine albanais : la phytonymie arbëreshe », Géolinguistique [En ligne], 18 | 2018, mis en ligne le 01 décembre 2018, consulté le 13 octobre 2021. URL : <http://journals.openedition.org/geolinguistique/329> ; DOI : $<$ https://journals.openedition.org/geolinguistique/329>

Ríos PANISSE Marìa del Carmine, 1983, Nomenclatura de la flora y fauna marítimas de Galicia, Santiago de Compostela, Universidad de Santiago de Compostela.

VENDRYÈs Joseph, LAMBERT Pierre-Yves, 1996, Lexique étymologique de l'irlandais ancien, (Lettre D), Paris, CNRS éd. Dublin, Dublin Institute for Advanced Studies (7 vol.). 
WAGNER Heinrich, Ó BAOILL Colm., 1958-1981, Linguistic Atlas and Survey of Irish Dialects, Dublin, Dublin Institute for Advanced Studies, 4 volumes.

\section{NOTES}

1. Depuis 2008 le Centre de Dialectologie de Grenoble a intégré le laboratoire GIPSA-lab, UMR 5216.

2. Atlas Linguistique des Côtes de l'Atlantique et de la Manche sous la direction de Daniel Le Bris.

3. Nous tenons à remercier les animatrices de l'EHPAD des Abers pour avoir facilité le bon déroulement des entretiens.

4. JP : Jean Pronost (1922-2008); VC: Vincent Coum (1907); YLG: Yves Le Goff : interrogés dans le cadre de l'ALCAM par Jean Ropars en mai 85. AmR: Ambroise Rouzig; AiR : Aimée Rouzig : interrogés par Mikael Madeg en 1980, Pitiod : Pd en 1977 et JP en 1990. ALB : Alain Le Berre, probablement informé par Jean Seite, responsable du comité local du goémon. PAB64 : article paru dans la revue Penn ar Bed en 1964 : «Le nom des algues en breton », vol. 34, p. 210. PT : ostréiculteur natif de Landéda. PAP : Pêcheur à pied, croisé à marée basse. YK : Yvonne Kerbrat (1928), Ar Vourc'h. MG : Madame Guillamoux (1939), Traoñ Bizin/traõ bi’zin/. Enquêtes personnelles réalisées en juillet 2021 à l'EHPAD des Abers.

5. Le gael. slat(a) mara désigne parfois le stipe de laminaire ou la laminaire dans sa totalité.

6. Toujours curieux, Kalm a goûté la zostère et a trouvé le goût agréable : « quand on la mâche, elle a un goût sucré et agréable ».

7. Désignation relevée par Agnes T. Brennan en 1950 auprès des habitants de Fenit/An Fhianait, comté de Kerry, Irlande.

8. National Folklore Collection, The Schools' Collection, Volume 0821, page 268.

9. Des tentatives de réduction des émissions de méthane sont envisagées par l'ajout de l'algue rouge au régime alimentaire des vaches.

10. Concordance motivationnelle avec la désignation galicienne pata de ghaliña, Fisterra, Dumbría, Carnota, Rianxo (Ríos, 1983).

\section{RÉSUMÉS}

La multiplication des études motivationnelles réalisées à partir de corpus régionaux (Lesbos, Arbëria, Scandinavie) nous amène à considérer la fréquence des classes motivationnelles dans l'espace comme nouveau critère d'analyse de la variation. Nous proposons de développer cette approche à partir du nom des algues en Basse-Bretagne (622 entrées) et en Irlande (1080). Si l'ensemble des classes motivationnelles sont identifiées (descriptives, zoomorphiques et anthropomorphiques), les motivations descriptives semblent prépondérantes.

Recent developments in motivational semantics based on dialectal material collected in Lesbos, Arbëria and Scandinavia suggest that motivational patterns can be considered as a new criterion for the study of variation. We choose to apply this method to a set of gathered and collected seaweed names in Lower-Brittany (622 entries) and Ireland (1080). The main motivational 
patterns are identified: descriptive, zoomorphic, anthropomorphic. Descriptive patterns seem to be more influential.

INDEX

Mots-clés : noms d'algues, motivation sémantique, isomotif, géolinguistique

Keywords : seaweed names, semantic motivation, isomotief, geolinguistics

\section{AUTEUR}

MAEL JÉZÉQUEL

Doctorant, Université de Bretagne occidentale, CRBC - Centre de recherche bretonne et celtique (EA 4451 / UMS 3554)

maeljezekel@gmail.com 